\title{
A Large-Displacement CMOS-Micromachined Thermal Actuator with Capacitive Position Sensing
}

\author{
Li-Sheng Zheng and Michael S.-C. Lu \\ Department of Electrical Engineering and Institute of Electronics Engineering \\ National Tsing Hua University \\ Hsinchu 30013, Taiwan, R.O.C. \\ sclu@ee.nthu.edu.tw
}

\begin{abstract}
In this paper, we present the design and characterization of a large-displacement thermal actuator fabricated in a conventional CMOS process. The thermallydriven microstructure is fabricated by two dry etching steps after the completion of CMOS. The structure contains multiple layers of metal, silicon dioxide, and polysilicon. To avoid drift caused by change of ambient temperature, we adopt a capacitive sensing scheme that uses vertically sensed comb electrodes with a nominal sensing capacitance of $40 \mathrm{fF}$. The microactuator is characterized by static and dynamic measurements, with a measured out-of-plane motion up to 24 $\mu \mathrm{m}$ at $17 \mathrm{~mW}$, a thermal time constant of $0.24 \mathrm{~ms}$, and a mechanical resonant frequency at $16.8 \mathrm{kHz}$. The measured minimum input-referred noise voltage of the sensing preamplifier is $5.9 \mu \mathrm{V} / \sqrt{ } \mathrm{Hz}$, equivalent to a minimum inputreferred noise displacement of $0.16 \mathrm{~nm} / \sqrt{ } \mathrm{Hz}$.
\end{abstract}

\section{INTRODUCTION}

Thermal actuation is known for its capability in producing a large and linear displacement with respect to heating power. The driving mechanisms include the wellknown bi-morph (or multi-morph) effect [1], or the use of hot- and cold arms [2]. In the latter, microstructures with a single-layer material can produce in-plane motion due to the difference in thermal expansion by use of a narrow "hot" arm and a wide "cold" arm. In either approach, the displacement of a thermal actuator is sensitive to the ambient operating temperature, which consequently limits its practical use for precise positioning. To overcome this disadvantage, a temperature-insensitive position sensing mechanism (e.g., capacitive sensing) is incorporated into our thermal actuator design.

Capacitive sensing is often preferred as the position sensing mechanism due to its high sensitivity. On-chip detection circuits are mostly required when a nominal sensing capacitance is much less than pico-farads, in order to enhance measured signal-to-noise ratio. The CMOS technology is an appropriate choice for implementation of capacitive sensing circuit, since MOS transistors provide high input impedances that match well with respect to the high-impedance capacitive signals. Only a few thermal actuators fabricated in CMOS provide motion detection; Lakdawala [3] reported a thermally stabilized CMOS accelerometer to provide a better dc bias stability and a reduced sensitivity variation due to structural curl. A thermally actuated cantilever with piezoresistive sensing was reported by Volden [4] for use in scanning force microscope.

This work will present the design and fabrication of a large-displacement CMOS-MEMS thermal actuator with integrated capacitive sensing. A large-displacement thermal actuator can be used as the shutter-type variable optical attenuator (VOA) [5,6] for optical communications, or implemented in an array format for micro manipulation [7]. The desired displacement is in the order of 10's of micrometers. The designed thermal actuator is a multi-layer structure. Vertical comb fingers are used in favor of the more sensitive parallel-plate capacitor to allow sensing of the desired large displacements. The experimental results show that a minimum input-referred noise displacement of 0.16 $\mathrm{nm} / \sqrt{ } \mathrm{Hz}$ can be achieved.

\section{DEVICE FABRICATION}

Fabrication of devices starts with the TSMC $0.35-\mu \mathrm{m}$ two-polysilicon four-metal CMOS process, followed by the post-CMOS micromachining steps similar to those described in [8]. First an anisotropic dielectric reactive-ion etch (RIE) with $\mathrm{CHF}_{3} / \mathrm{O}_{2}$ plasma defines the structural sidewalls with the top metal layer used as an etch-resistant mask. The achieved oxide etch rate is $500 \AA / \mathrm{min}$. Next we perform an isotropic silicon etch with $\mathrm{XeF}_{2}$ for structural release at an etch rate of $0.5 \mu \mathrm{m} / \mathrm{min}$. The released structure thus contains multiple metal and dielectric layers, and can use the polysilicon layer as the heater. Removal of the silicon allows us to design a large-displacement thermal actuator that moves toward the substrate when heated. The CMOS sensing circuit can be placed near the etched pit to give a small routing capacitance, leading to a better sensing capability. 


\section{CMOS-MEMS THERMAL ACTUATOR}

\section{A. Mechanical Design}

As shown in Fig. 1, we intend to fabricate a 1-D VOA array for equalization of the transmitted light signals. The out-of-plane cantilever displacement produced by thermal actuation moves the micro-mirrors on top of those microstructures to block or pass the light in a continuous fashion. Fabrication of the micro-mirrors requires an additional lithographic step for electroplating before the dryetching steps described earlier. The required displacement is $20 \mu \mathrm{m}$ to allow a complete pass or block of light. To ensure accurate positioning, temperature-insensitive capacitive sensing is incorporated for operations under various thermal conditions. While achieving the maximal displacement, the heating temperature should not exceed the limit that CMOS transistors and metallization can tolerate.

The original analysis of a bi-morph cantilever can be found in [9]. As for multi-morph structures, DeVoe et al. presented the analysis of piezoelectric cantilevers [10]. Following that, the analysis of a CMOS-MEMS thermal actuator was reported by Lakdawala [11]. For cantilevers composed of layers of the same length, the resulting radius of curvature $\rho$ with respect to the temperature change could be written as:

$$
T-T_{o}=\frac{1}{\rho}\left(\frac{2 \chi-Y^{T} G^{-1} S}{Y^{T} G^{-1} A}\right)
$$

where $T$ is the characteristic temperature, $T_{o}$ is the reference temperature, $\chi$ is the total flexural rigidity of the beam, the matrix $\boldsymbol{G}$ describes the effects of width, thickness, and the Young's modulus, the matrices $\boldsymbol{Y}$ and $\boldsymbol{S}$ describe the thickness effects, and the matrix $\boldsymbol{A}$ describes the effect of thermal coefficient of expansion (TCE).

To achieve a large displacement, the current design contains three metal layers (metal-1 to metal-3) without inclusion of the top metal-4 in order to reduce the out-ofplane rigidity. The structure is $300 \mu \mathrm{m}$ in length and $17 \mu \mathrm{m}$ in width. The polysilicon heater $(8 \Omega / \mathrm{sq}$.) is routed from the anchor to about two-thirds of the structural length. The rest of the structure without the polysilicon provides further amplification of the cantilever motion. The design has comb electrodes on both sides of the free end for capacitive detection of the vertical displacement between rotor and stator electrodes. The capacitive sensitivity is enhanced by having a narrow gap between sensing electrodes. The electrodes have vias filled around the peripheral in between metal layers in order to form metal-to-metal capacitances. The current design has a finger overlap of $50 \mu \mathrm{m}$, a gap of 2 $\mu \mathrm{m}$, and a total of 18 rotor fingers to give a nominal sensing capacitance of $51 \mathrm{fF}$. The micrograph of the fabricated actuator is shown in Fig. 2. R.O.C.

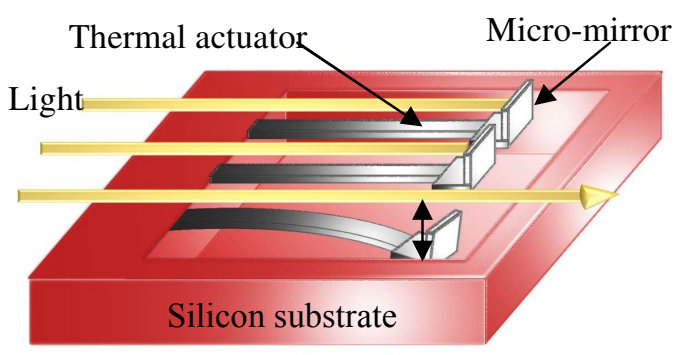

Figure 1. 1-D VOA array implemented by CMOS-MEMS thermal actuators

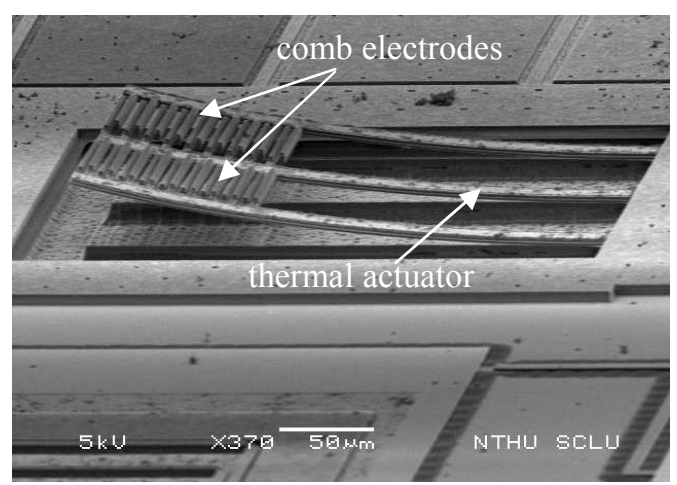

Figure 2. SEM of the fabricated actuator with sensing electrodes.

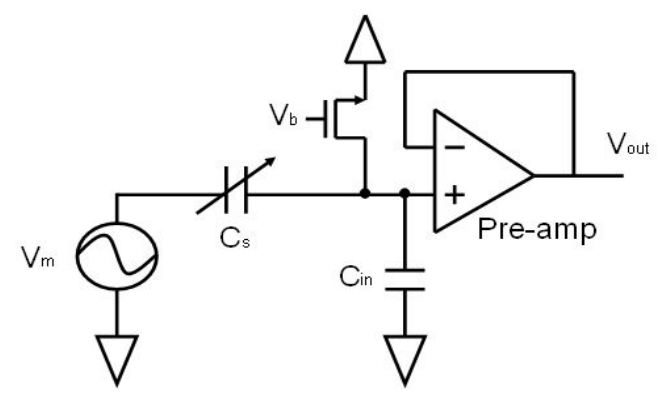

Figure 3. Schematic of the single-ended capacitive sensing circuit.

\section{B. Capacitive Sensing Circuit}

The schematic of the capacitive sensing circuit is shown in Fig. 3, in which the single-ended signal is taken from the mid-point between the sensing capacitance and the pre-amp input capacitance. The pre-amp is a unity-gain buffer implemented by a two-stage operational amplifier, with PMOS input transistors intended for reduction of the flicker noise at low frequencies. By simulation, the op-amp achieves an open-loop gain of $69 \mathrm{~dB}$, a unity-gain frequency of 12.7 $\mathrm{MHz}$, and a phase margin of $60^{\circ}$. The d.c. bias at the input sensing node is provided by a transistor operated in the subthreshold region. 


\section{EXPERIMENT}

The measured resistance of the polysilicon heater was $995 \Omega$, as compared to $803 \Omega$ obtained from circuit extraction. Temperature distribution of the heated microstructure for each applied voltage was obtained by infrared measurements (Infrascope II from QFI). The highest temperature occurred near the end of the heating resistor and was measured around $232^{\circ} \mathrm{C}$ at an applied power of $17 \mathrm{~mW}$, close to $245^{\circ} \mathrm{C}$ obtained by the finiteelement simulation. Fig. 4 shows the measured resistor values with respect to the temperature, indicating a temperature coefficient of resistance (TCR) of $0.1 \% / \mathrm{K}$. The actuated displacement at the free end was measured by a 3$\mathrm{D}$ optical profiler with respect to the applied voltage. The displacement and input power displays a linear relationship as shown in Fig. 5, with a maximum displacement of $24 \mu \mathrm{m}$ toward the substrate at $17 \mathrm{~mW}$.

The dynamic response of the actuator in the out-of-plane motion was measured by a Laser Doppler Vibrometer (LDV). The measured resonant frequency is $16.8 \mathrm{kHz}$ with a quality factor of 21 . The bandwidth of the sensing preamp was measured at $12.1 \mathrm{MHz}$ by an Agilent 4395A network/spectrum analyzer, closed to the simulated value of 12.7 MHz. The measured frequency response of a test circuit leads to an extracted preamp input capacitance at 55 $\mathrm{fF}$, close to the value of $45 \mathrm{fF}$ from circuit simulation. Based on the measured input capacitance, we subsequently obtained the initial sensing capacitance of the actuator at 40 $\mathrm{fF}$, which is less than the calculated value of $51 \mathrm{fF}$ due to overlap mismatch between the sensing electrodes. The preamp signal was modulated at $10.5 \mathrm{MHz}$ in order to avoid the flicker noise, with the magnitude being measured by a spectrum analyzer at a $10-\mathrm{Hz}$ resolution with respect to the actuated displacement, as shown in Fig. 6. The minimum input-referred noise voltage was measured at $5.9 \mu \mathrm{V} / \sqrt{ } \mathrm{Hz}$. Fig. 7 shows the measured sensitivity at each displacement, which leads to a minimum input-referred noise displacement of $0.16 \mathrm{~nm} / \sqrt{ } \mathrm{Hz}$ at a displacement of $8.75 \mu \mathrm{m}$. The drop of sensitivity at large displacements is due to the non-overlap of sensing electrodes, which will be solved in the future by adding thermal actuation capability to the stator electrodes so as to maintain a constant sensitivity.

The transient response of the actuator in Fig. 8 was measured at the preamp output with an applied square-wave heating voltage at $200 \mathrm{~Hz}$. The envelope of the sensed signal leads to a thermal time constant of $0.24 \mathrm{~ms}$, equivalent to a pole frequency of $4167 \mathrm{rad} / \mathrm{sec}$ for a first-order linear system.

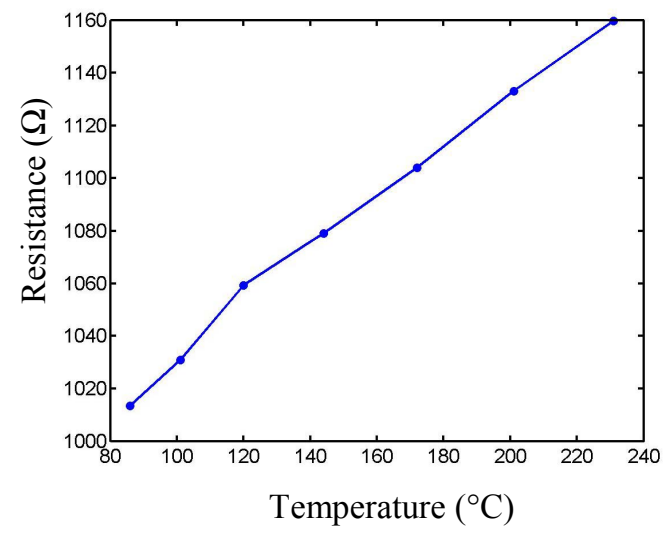

Figure 4. Measured polysilicon resistance vs. temperature.

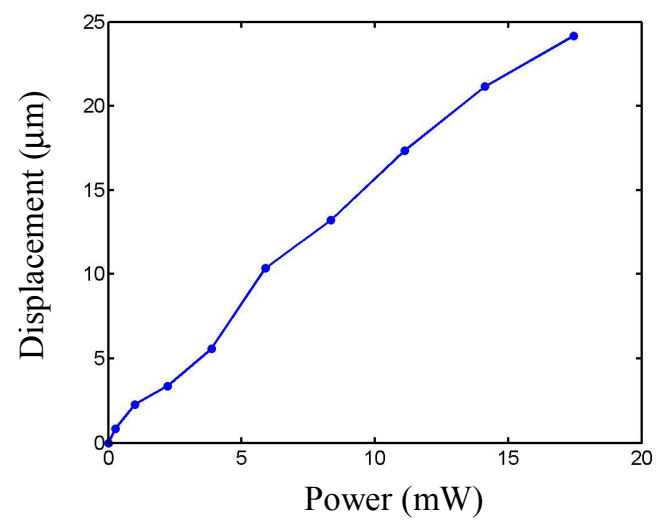

Figure 5. Measured displacement vs. applied power.

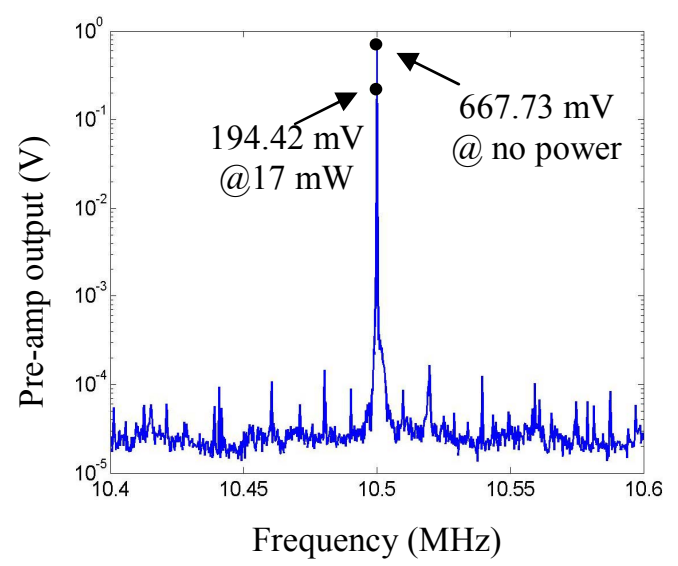

Figure 6. Modulated pre-amp output measured from a spectrum analyzer. 


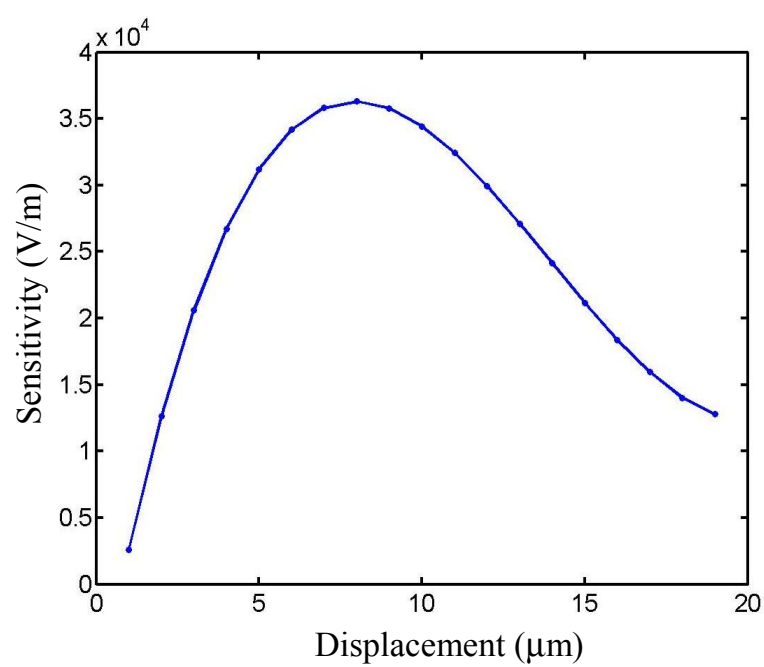

Figure 7. Measured capacitive sensitivity at each displacement.

Square-wave heating voltage at $200 \mathrm{~Hz}$

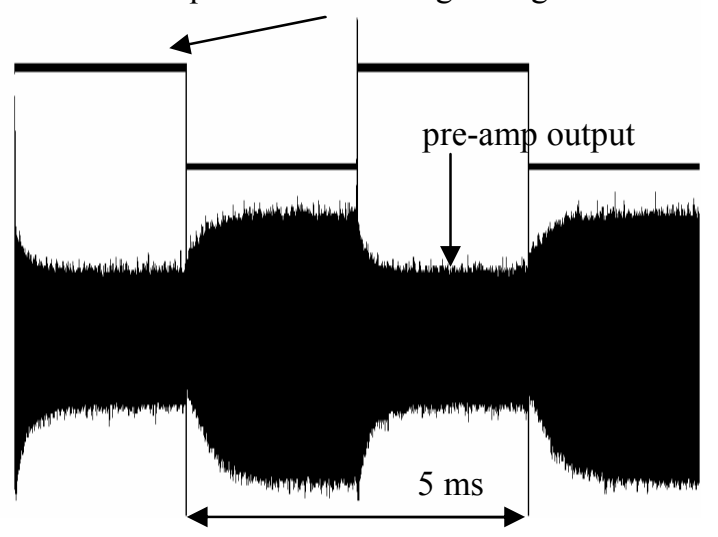

Figure 8. Pre-amp output to a $200-\mathrm{Hz}$ square-wave heating voltage, illustrating that the thermal time constant of the actuator is $0.24 \mathrm{~ms}$.

\section{CONCLUSION}

This work presents a multi-morph thermal actuator fabricated in a conventional CMOS process, with combfinger electrodes being used to allow capacitive position detection with respect to large displacements. The experimental results show that a $24-\mu \mathrm{m}$ out-of-plane displacement is achieved at $17 \mathrm{~mW}$ with a maximum temperature occurred at $232{ }^{\circ} \mathrm{C}$. The minimum input-referred noise displacement of the sensing pre-amp is $0.16 \mathrm{~nm} / \sqrt{\mathrm{Hz}}$. For a closed-loop control bandwidth at $1 \mathrm{kHz}$, the sensor noise will limit the minimum detectable signal to $5 \mathrm{~nm}$, which is enough for the VOA array application.

\section{ACKNOWLEDGMENT}

This research effort is sponsored by the National Science Council, Taiwan, R.O.C. (NSC 94-2215-E-007-020). The authors would like to thank the National Chip Implementation Center of the National Applied Research Laboratories for support of CMOS fabrication, and Prof. Weileun Fang and Prof. Da-Jeng Yao for use of their laboratory resources.

\section{REFERENCES}

[1] M. Ataka, A. Omodaka, and J. Fujita, "A biomimetic micro motion system," Dig. $7^{\text {th }}$ Int. Conf. on Solid State Sensors and Actuators (Transducers '93), pp. 38-41, Yokohama, Japan, June 1993.

[2] C. Marxer, P. Griss, and N. F. de Rooij, "A variable optical attenuator based on silicon micromechanics," IEEE Photonics Tech. Lett., vol. 11, no. 2, pp. 233-235, Feb. 1999.

[3] H. Lakdawala and G. Fedder, "Temperature stabilization of CMOS capacitive accelerometers," J. Micromech. and Microeng., vol. 14, no. 4, pp. 559-566, 2004.

[4] T. Volden, M. Zimmermann, D. Lange, O. Brand, and H. Baltes, "Dynamics of CMOS-based thermally actuated cantilever arrays for force microscopy," Sensors and Actuators A, vol. 115, pp. 516-522, 2004.

[5] R. Syms, H. Zou, J. Stagg, and H. Veladi, "Sliding-blade MEMS iris and variable optical attenuator," J. Micromech. and Microeng., vol. 14, no. 12, pp. 1700-1710, Dec. 2004.

[6] R. Wood, V. Dhuler, and E. Hill, "A MEMS variable optical attenuator," IEEE/LEOS Int. Conf. on Optical MEMS, pp. 121-122, Aug. 2000.

[7] J. W. Suh, R. B. Darling, K.-F. Böhringer, B. R. Donald, H. Baltes, G. T. A. Kovacs, "CMOS integrated ciliary actuator array as a generalpurpose micromanipulation tool for small objects," $J$. Microelectromech. Syst., vol. 8, no. 4, pp. 483-496, Dec. 1999.

[8] G. K. Fedder, S. Santhanam, M. L. Reed, S. C. Eagle, D. F. Guillou, M. S.-C. Lu, and L. R. Carley, "Laminated high-aspect-ratio microstructures in a conventional CMOS process," Sensors and Actuators A (Physical), vol. A57, no. 2, pp. 103-110, 1997.

[9] S. Timoshenko, "Analysis of bi-metal thermostats," J. Optical Soc. of America, vol. 11, pp. 233-255, 1925.

[10] D. L. DeVoe and A. P. Pisano, "Modeling and optimal design of piezoelectric cantilever microactuators," J. Microelectromech. Syst., vol. 6, no. 3, pp. 266-270, Sept. 1997.

[11] H. Lakdawala and G. Fedder, "Analysis of temperature-dependent residual stress gradients in CMOS micromachined structures," in Tech. Digest of the $10^{\text {th }}$ Int. Conf. on Solid-State Sensors and Actuators (Transducers '99), pp. 526-529, Sendai, Japan, June 7-10, 1999. 